\title{
Efficient Cross-Coupling of Functionalized Arylzinc Halides Catalyzed by a Nickel Chloride-Diethyl Phosphite System
}

\author{
Andrei Gavryushin, Christiane Kofink, Georg Manolikakes and Paul Knochel* \\ Department Chemie, Ludwig-Maximilians-Universität, Butenandtstrasse 5-13, 81377, München (Germany). \\ Paul.Knochel@cup.uni-muenchen.de
}

\section{Supporting Information}

\section{General considerations}

Unless otherwise indicated, all reactions were carried out with magnetic stirring and in case of air- or moisture-sensitive compounds in flame-dried glassware under argon. Syringes were used to transfer the reagents and the solvents were purged with argon prior to use. Reactions were monitored by gas chromatography (GC and GC-MS) or thin layer chromatography.

Solutions of organomagnesium compounds were prepared, if not especially mentioned, by the reaction of $\mathrm{Mg}$ with aryl bromides in THF, titrated with a standard solution of $\mathrm{I}_{2}$ in $0.5 \mathrm{M}$ $\mathrm{LiCl}$ in THF and diluted with THF to the mentioned concentration. $\mathrm{ZnBr}_{2}$ and $\mathrm{ZnCl}_{2}$ were dried at $140{ }^{\circ} \mathrm{C}$ under high vacuum for $30 \mathrm{~min}$ and then dissolved in dry THF.

\section{Typical Procedure 1 (TP 1).}

The solution of the nickel catalyst was prepared as follows. In a $25 \mathrm{~mL}$ Schlenk tube under argon in dry degassed N-ethylpyrrolidinone $(10.0 \mathrm{~mL})$ were dissolved anhydrous $\mathrm{NiCl}_{2}(8.2$ $\mathrm{mg}, 0.063 \mathrm{mmol}),(\mathrm{EtO})_{2} \mathrm{P}(\mathrm{O}) \mathrm{H}(34.5 \mathrm{mg}, 0.25 \mathrm{mmol})$ and DMAP (30.5 mg, $\left.0.25 \mathrm{mmol}\right)$.

In a dry and argon flushed $25 \mathrm{~mL}$ flask, equipped with a magnetic stirrer and a septum, the corresponding arylmagnesium reagent in THF $(1.20 \mathrm{mmol})$ was added slowly with cooling to the solution of $\mathrm{ZnBr}_{2}(0.67 \mathrm{~mL}$ of $1.5 \mathrm{M}$ in THF, $1.00 \mathrm{mmol})$ and NEP $(0.17 \mathrm{~mL})$. To this mixture, the electrophile (aryl halide or sulfonate, $1.00 \mathrm{mmol}$ ) was added, followed by the solution of the catalyst $(0.08 \mathrm{~mL})$. The final THF-NEP volume ratio should be approximately 8:1. The mixture was stirred at the specified temperature until the GC of an aliquot showed the reaction completion, quenched with sat. $\mathrm{NH}_{4} \mathrm{Cl}$ solution, extracted with ether, and the product was purified by column chromatography. 


\section{3-Fluoro-4'-methoxy-1,1'-biphenyl (3a):}

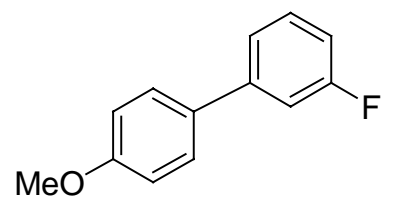

Prepared according to the TP 1 . To the $\mathrm{ZnBr}_{2}$ solution $(0.67 \mathrm{~mL}, 1.5 \mathrm{M}$ in THF) and NEP $(0.17 \mathrm{~mL})$ was added dropwise 4-methoxyphenylmagnesium bromide $(1.57 \mathrm{~mL}, 0.83 \mathrm{M}$ in $\mathrm{THF})$, then the catalyst solution $(0.08 \mathrm{~mL})$ and 3-bromofluorobenzene $(175 \mathrm{mg}, 1.00 \mathrm{mmol})$. Stirred $2 \mathrm{~h}$ at RT. The usual workup and purification by flash chromatography (pentane/ether 19:1) yielded 3a as white solid (174 $\mathrm{mg}, 86 \%)$.

Mp.: $67-67.5^{\circ} \mathrm{C}$. (Lit. ${ }^{1}: 68^{\circ} \mathrm{C}$ )

${ }^{1} \mathbf{H}$-NMR $\left(\mathrm{CDCl}_{3}, 300 \mathrm{MHz}, 25^{\circ} \mathrm{C}\right): \delta=7.39(\mathrm{~d}, J=8.9 \mathrm{~Hz}, 2 \mathrm{H}), 7.28-7.11(\mathrm{~m}, 3 \mathrm{H}), 6.90-$ $6.84(\mathrm{~m}, 3 \mathrm{H}), 3.72(\mathrm{~s}, 3 \mathrm{H})$.

${ }^{13} \mathrm{C}-\mathrm{NMR}\left(\mathrm{CDCl}_{3}, 75 \mathrm{MHz}, 25{ }^{\circ} \mathrm{C}\right): \delta=163.2\left(\mathrm{q},{ }^{1} \mathrm{~J}(\mathrm{C}, \mathrm{F})=245 \mathrm{~Hz}\right), 159.5,143.1\left(\mathrm{q},{ }^{3} J(\mathrm{C}\right.$, $\mathrm{F})=7.6 \mathrm{~Hz}), 132.4\left(\mathrm{q},{ }^{4} J(\mathrm{C}, \mathrm{F})=2.1 \mathrm{~Hz}\right), 130.1\left(\mathrm{q},{ }^{3} J(\mathrm{C}, \mathrm{F})=8.2 \mathrm{~Hz}\right), 128.1,122.2\left(\mathrm{q},{ }^{4} J(\mathrm{C}\right.$, $\mathrm{F})=2.6 \mathrm{~Hz}), 114.5,113.5\left(\mathrm{q},{ }^{2} J(\mathrm{C}, \mathrm{F})=21.7 \mathrm{~Hz}\right), 113.3\left(\mathrm{q},{ }^{2} J(\mathrm{C}, \mathrm{F})=21.1 \mathrm{~Hz}\right), 55.3$.

IR (KBr): 2963 (w), 2840 (w), 1610 (vs), 1589 (s), 1573 (m), 1522 (s), 1487 (s), 1447 (m), 1292 (s), 1264 (s), 1252 (s), 1189 (vs), 1162 (m), 1026 (m), 879 (m), 830 (vs), 782 (s).

MS (70 eV, EI), m/z (\%): 209 (100, M+), 187 (50), 159 (54), 133 (24), 107 (10), 77 (13).

HRMS $m / z$ : calcd. for $\mathrm{C}_{13} \mathrm{H}_{11} \mathrm{FO}: 202.0794$; found: 202.0790 .

\section{Ethyl 4'-methoxy-biphenyl-3-carboxylate (3b)}

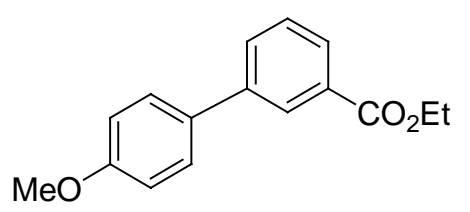

Prepared according to the TP 1 . To the $\mathrm{ZnBr}_{2}$ solution $(0.67 \mathrm{~mL}, 1.5 \mathrm{M}$ in THF) and NEP $(0.17 \mathrm{~mL})$ was added dropwise 4-methoxyphenylmagnesium bromide $(1.57 \mathrm{~mL}, 0.83 \mathrm{M}$ in THF), then the catalyst solution $(0.08 \mathrm{~mL})$, and ethyl 3-bromobenzoate (229 mg, $1.00 \mathrm{mmol})$. Stirred for $1 \mathrm{~h}$ at RT. The usual workup and purification by flash chromatography (pentane/ether 9:1) yielded $\mathbf{3 b}$ as colourless oil (234 mg, 91\%). 
${ }^{1} \mathbf{H}-\mathbf{N M R}\left(\mathrm{CDCl}_{3}, 300 \mathrm{MHz}, 25^{\circ} \mathrm{C}\right): \delta=8.26(\mathrm{~s}, 1 \mathrm{H}), 8.00-7.97(\mathrm{~m}, 1 \mathrm{H}), 7.73-7.70(\mathrm{~m}, 1 \mathrm{H})$, $7.56(\mathrm{~d}, J=8.8 \mathrm{~Hz}, 2 \mathrm{H}), 7.46(\mathrm{t}, J=7.7 \mathrm{~Hz}, 1 \mathrm{H}), 6.99(\mathrm{~d}, J=8.7 \mathrm{~Hz}, 2 \mathrm{H}), 4.41(\mathrm{q}, J=7.1 \mathrm{~Hz}$, $2 \mathrm{H}), 3.83(\mathrm{~s}, 3 \mathrm{H}), 1.41(\mathrm{t}, J=7.1 \mathrm{~Hz}, 3 \mathrm{H})$.

${ }^{13} \mathrm{C}-\mathrm{NMR}\left(\mathrm{CDCl}_{3}, 75 \mathrm{MHz}, 25{ }^{\circ} \mathrm{C}\right): \delta=166.5,159.4,140.9,132.5,130.9,130.8,128.6$, 128.1, 127.6, 127.1, 114.2, 60.9, 55.2, 14.2.

IR (KBr): 2981 (w), 1717 (vs), 1610 (m), 1518 (s), 1439 (m), 1367 (w), 1300 (s), 1249 (vs), 1182 (m), 1109 (s), 1049 (m), 1030 (m), 834 (m), 758 (s), $574(\mathrm{w})$.

MS (70 eV, EI), m/z (\%): $256\left(100, \mathrm{M}^{+}\right), 241$ (9), 228 (11), 211 (20), 183 (10), 168 (6), 139 (12), 105 (3).

HRMS $m / z$ : calcd. For $\mathrm{C}_{16} \mathrm{H}_{16} \mathrm{O}_{3}: 256.1099$; found: 256.1097 .

Ethyl 4'-methoxy-biphenyl-4-carboxylate (3c)

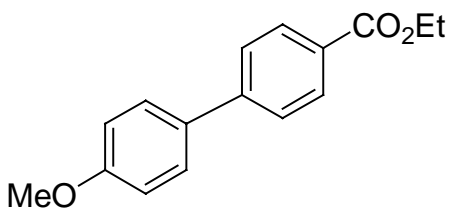

Prepared according to the TP 1 . To the $\mathrm{ZnBr}_{2}$ solution $(0.67 \mathrm{~mL}, 1.5 \mathrm{M}$ in THF) and NEP $(0.17 \mathrm{~mL})$ was added dropwise 4-methoxyphenylmagnesium bromide $(1.57 \mathrm{~mL}, 0.83 \mathrm{M}$ in THF), then the catalyst solution $(0.08 \mathrm{~mL})$ and ethyl 4-bromobenzoate $(229 \mathrm{mg}, 1.00 \mathrm{mmol})$ or ethyl 4-chlorobenzoate $(185 \mathrm{mg}, 1.00 \mathrm{mmol})$. The mixture was stirred for $1 \mathrm{~h}$ at RT $(48 \mathrm{~h}$ for ethyl 4-chlorobenzoate). The usual workup and purification by flash chromatography (pentane-ether 9:1) yielded 3c as white solid (224 mg or 87\% for the reaction with ethyl 4bromobenzoate and $214 \mathrm{mg}$ or $83 \%$ for ethyl 4-chlorobenzoate). The analytical data are in accordance with the literature. ${ }^{3}$

Mp.: $100-101^{\circ} \mathrm{C}$.

${ }^{1}$ H-NMR $\left(\mathrm{CDCl}_{3}, 300 \mathrm{MHz}, 25{ }^{\circ} \mathrm{C}\right): \delta=8.09(\mathrm{~d}, J=8.7 \mathrm{~Hz}, 2 \mathrm{H}), 7.62-7.55(\mathrm{~m}, 4 \mathrm{H}), 6.99(\mathrm{~d}$, $J=8.7 \mathrm{~Hz}, 2 \mathrm{H}), 4.39(\mathrm{q}, J=7.1 \mathrm{~Hz}, 2 \mathrm{H}), 3.84(\mathrm{~s}, 3 \mathrm{H}), 1.41(\mathrm{t}, J=7.1 \mathrm{~Hz}, 3 \mathrm{H})$.

${ }^{13} \mathrm{C}-\mathrm{NMR}\left(\mathrm{CDCl}_{3}, 75 \mathrm{MHz}, 25{ }^{\circ} \mathrm{C}\right): \delta=166.5,159.8,145.0,132.4,130.0,128.6,128.3$, $126.4,114.3,60.8,55.3,14.3$. 


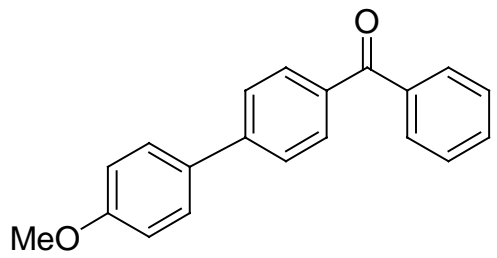

Prepared according to the TP 1 . To the $\mathrm{ZnBr}_{2}$ solution $(0.67 \mathrm{~mL}, 1.5 \mathrm{M}$ in THF) and NEP $(0.17 \mathrm{~mL})$ was added dropwise 4-methoxyphenylmagnesium bromide $(1.57 \mathrm{~mL}, 0.83 \mathrm{M}$ in $\mathrm{THF})$, then the catalyst solution $(0.08 \mathrm{~mL})$ and 4-bromobenzophenone (261 $\mathrm{mg}, 1.00 \mathrm{mmol})$. Stirred for $3 \mathrm{~h}$ at RT. The usual workup and purification by flash chromatography (pentaneether 19:1) yielded $\mathbf{3 c}$ as a white solid (210 $\mathrm{mg}, 73 \%)$. The analytical data are in accordance with the literature. ${ }^{2}$

Mp.: $167-168^{\circ} \mathrm{C}$.

${ }^{1} \mathbf{H}-\mathbf{N M R}\left(\mathrm{CDCl}_{3}, 600 \mathrm{MHz}, 25{ }^{\circ} \mathrm{C}\right): \delta=7.87(\mathrm{~d}, J=8.1 \mathrm{~Hz}, 2 \mathrm{H}), 7.83(\mathrm{~d}, J=8.3 \mathrm{~Hz}, 2 \mathrm{H})$, $7.66(\mathrm{~d}, J=8.3 \mathrm{~Hz}, 2 \mathrm{H}), 7.60-7.57(\mathrm{~m}, 3 \mathrm{H}), 7.49(\mathrm{t}, J=7.6 \mathrm{~Hz}, 2 \mathrm{H}), 7.01(\mathrm{~d}, J=8.8 \mathrm{~Hz}, 2 \mathrm{H})$, $3.86(\mathrm{~s}, 3 \mathrm{H})$.

${ }^{13} \mathrm{C}-\mathrm{NMR}\left(\mathrm{CDCl}_{3}, 151 \mathrm{MHz}, 25{ }^{\circ} \mathrm{C}\right): \delta=196.3,159.9,144.8,137.9,135.6,132.4,132.2$, $130.8,129.9,128.4,128.3,126.4,114.4,55.4$.

IR (KBr): 1651 (vs), 1600 (vs), 1529 (w), 1446 (w), 1316 (m), 1288 (s), 1276 (s), 1256 (m), 1206 (vs), 1182 (w), 1033 (w), 939 (w), 829 (s), 794 (w), 697 (m).

MS (70 eV, EI), m/z (\%): $288\left(100, \mathrm{M}^{+}\right), 211$ (76), 183 (6), 168 (8), 139 (8), 105 (11), 77 (10), $51(1)$.

HRMS $m / z$ : calcd. for $\mathrm{C}_{20} \mathrm{H}_{16} \mathrm{O}_{2}: 288.1150$; found: 288.1146 .

\section{3-(4-Methoxyphenyl)-pyridine (3e)}

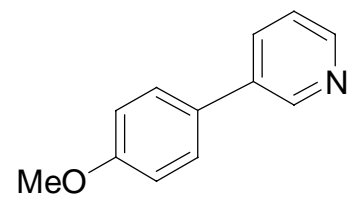

Prepared according to the TP 1 . To the $\mathrm{ZnBr}_{2}$ solution $(0.67 \mathrm{~mL}, 1.5 \mathrm{M}$ in THF) and NEP $(0.17 \mathrm{~mL})$ was added dropwise 4-methoxyphenylmagnesium bromide $(1.57 \mathrm{~mL}, 0.83 \mathrm{M}$ in 
THF), then the catalyst solution $(0.08 \mathrm{~mL})$ and 3-bromopyridine $(159 \mathrm{mg}, 1.00 \mathrm{mmol})$ or 3Chloro-pyridine (114 mg, $1.00 \mathrm{mmol})$. Stirred for $2 \mathrm{~h}$ at RT (12 $\mathrm{h}$ for 3-chloropyridine). The usual workup and purification by flash chromatography (pentane-ether 1:1) yielded $\mathbf{3 e}$ as a white solid (150 mg or $81 \%$ for 3-bromopyridine and $126 \mathrm{mg}$, $68 \%$ for 3-chloropyridine). The analytical data are in accordance with the literature. ${ }^{4}$ The same reaction performed on $20 \mathrm{mmol}$ scale gave the yield of $82 \%$.

Mp.: $62-63{ }^{\circ} \mathrm{C}$.

${ }^{1} \mathbf{H}$-NMR $\left(\mathrm{CDCl}_{3}, 600 \mathrm{MHz}, 25^{\circ} \mathrm{C}\right): \delta=8.81-8.80(\mathrm{~m}, 1 \mathrm{H}), 8.53\left(\mathrm{dd}, J_{1}=4.8 \mathrm{~Hz}, J_{2}=1.6\right.$ $\mathrm{Hz}, 1 \mathrm{H}), 7.84-7.80(\mathrm{~m}, 1 \mathrm{H}), 7.52(\mathrm{~d}, J=8.8 \mathrm{~Hz}, 2 \mathrm{H}), 7.34-7.30(\mathrm{~m}, 1 \mathrm{H}), 7.01(\mathrm{~d}, J=8.8 \mathrm{~Hz}$, $2 \mathrm{H}), 3.85(\mathrm{~s}, 3 \mathrm{H})$.

${ }^{13} \mathrm{C}-\mathrm{NMR}\left(\mathrm{CDCl}_{3}, 151 \mathrm{MHz}, 25{ }^{\circ} \mathrm{C}\right): \delta=159.7,148.0,147.9,136.3,133.8,130.3,128.2$, $123.5,114.6,55.4$.

IR (KBr): 2964 (w), 1608 (s), 1578 (w), 1564 (w), 1520 (s), 1478 (s), 1434 (m), 1283 (s), 1254 (vs), 1183 (s), 1030 (s), 838 (m), 803 (vs), 706 (m), 619 (w), 552 (w).

MS (70 eV, EI), m/z (\%): 185 (100, M+), 170 (44), 142 (46), 115 (17), 89 (11), 63 (8).

HRMS $m / z$ : calcd. for $\mathrm{C}_{12} \mathrm{H}_{11} \mathrm{NO}: 185.0841$; found: 185.0837 .

\section{6-(3-Methoxy-phenyl)-nicotinic acid methyl ester (3f)}

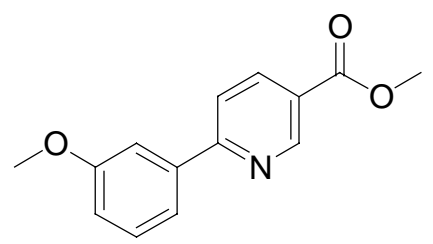

Prepared according to the TP 1 . To the $\mathrm{ZnBr}_{2}$ solution $(0.67 \mathrm{~mL}, 1.5 \mathrm{M}$ in THF) and NEP (0.17 mL) was added dropwise 3-methoxy-magnesium bromide (1.57 mL, 0.83 $\mathrm{M}$ in THF), then the catalyst solution $(0.08 \mathrm{~mL}$ in $\mathrm{NEP})$ and 6-chloronicotinic acid methyl ester $(172 \mathrm{mg}$, $1.00 \mathrm{mmol}$ ). Stirred for $24 \mathrm{~h}$ at RT, standard workup and purification by flash chromatography $\left(\mathrm{CH}_{2} \mathrm{Cl}_{2}\right.$-pentane 1:1) yielded $\mathbf{3 f}$ as colourless solid (180 mg, 74\%). Mp.: 89.5-90 ${ }^{\circ} \mathrm{C}$.

${ }^{1} \mathrm{H}-\mathrm{NMR}\left(\mathrm{CDCl}_{3}, 600 \mathrm{MHz}, 25^{\circ} \mathrm{C}\right): \delta=9.24(\mathrm{~s}, 1 \mathrm{H}), 8.31\left(\mathrm{dd}, J_{l}=8.3 \mathrm{~Hz}, J_{2}=1.9 \mathrm{~Hz}, 1 \mathrm{H}\right)$, $7.77(\mathrm{~d}, J=8.3 \mathrm{~Hz}, 1 \mathrm{H}), 7.63-7.62(\mathrm{~m}, 1 \mathrm{H}), 7.57(\mathrm{~d}, J=7.9 \mathrm{~Hz}, 1 \mathrm{H}), 7.38(\mathrm{t}, J=8.1 \mathrm{~Hz}, 1 \mathrm{H})$, $6.99\left(\mathrm{dd}, J_{1}=8.1 \mathrm{~Hz}, J_{2}=2.4 \mathrm{~Hz}, 1 \mathrm{H}\right), 3.94(\mathrm{~s}, 3 \mathrm{H}), 3.88(\mathrm{~s}, 3 \mathrm{H})$. 
${ }^{13} \mathbf{C}-\mathbf{N M R}\left(\mathrm{CDCl}_{3}, 151 \mathrm{MHz}, 25{ }^{\circ} \mathrm{C}\right): \delta=165.9,160.7,160.2,151.0,139.7,137.9,129.9$, $124.3,120.0,119.7,116.1,112.5,55.4,52.3$.

IR (KBr): 3059 (w), 3013 (w), 2954 (m), 2925 (m), 1715 (vs), 1596 (vs), 1562 (m), 1480 (s), 1433 (s), 1288 (vs), 1267 (s), 1231 (s), 1117 (s), 1030 (s), 1021 (s).

MS (70 eV, EI), m/z (\%): 243 (65, M+), 242 (100), 213 (38), 182 (9), 154 (10), 106 (11).

HRMS $m / z$ : calcd. for $\mathrm{C}_{14} \mathrm{H}_{13} \mathrm{NO}_{3}$ : 243.0895; found: 243.0867 .

\section{1-(3'-Methoxy-biphenyl-4-yl)-ethanone (3g).}

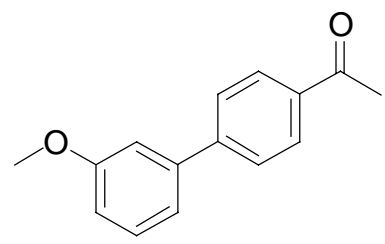

Prepared according to the TP 1. To the $\mathrm{ZnBr}_{2}$ solution $(0.67 \mathrm{~mL}, 1.5 \mathrm{M}$ in THF) and NEP $(0.17 \mathrm{~mL})$ was added dropwise 3-methoxy-magnesium bromide $(1.57 \mathrm{~mL}, 0.83 \mathrm{M}$ in $\mathrm{THF})$, then the catalyst solution $(0.08 \mathrm{~mL})$ and 4-bromoacetophenone $(199 \mathrm{mg}, 1.00 \mathrm{mmol})$. Stirred $2.5 \mathrm{~h}$ at RT. The standard workup and purification by flash chromatography $\left(\mathrm{CH}_{2} \mathrm{Cl}_{2}\right.$-pentane 1:1) yielded $\mathbf{3 g}$ as a yellow solid (175 $\mathrm{mg}, 77 \%)$.

Mp.: $35-36{ }^{\circ} \mathrm{C} .{ }^{5}$

${ }^{1} \mathbf{H}-\mathbf{N M R}\left(\mathrm{CDCl}_{3}, 300 \mathrm{MHz}, 25{ }^{\circ} \mathrm{C}\right): \delta=8.00\left(\mathrm{ddd}, J_{1}=8.5 \mathrm{~Hz}, J_{2}=2.9 \mathrm{~Hz}, J_{3}=1.9 \mathrm{~Hz}, 2 \mathrm{H}\right)$, $7.65\left(\mathrm{ddd}, J_{l}=8.6 \mathrm{~Hz}, J_{2}=2.0 \mathrm{~Hz}, J_{3}=1.9 \mathrm{~Hz}, 2 \mathrm{H}\right), 7.38-7.31(\mathrm{~m}, 1 \mathrm{H}), 7.20-7.17(\mathrm{~m}, 1 \mathrm{H})$, 7.13-7.12 (m, 1H), 6.94-6.90 (m, 1H), $3.85(\mathrm{~s}, 3 \mathrm{H}), 2.61(\mathrm{~s}, 3 \mathrm{H})$.

${ }^{13} \mathrm{C}-\mathrm{NMR}\left(\mathrm{CDCl}_{3}, 75 \mathrm{MHz}, 25{ }^{\circ} \mathrm{C}\right): \delta=198.1,160.4,146.0,141.8,136.4,130.4,129.3$, 127.7, 120.1, 113.9, 113.5, 55.8, 27.0.

MS (70 eV, EI), m/z (\%): $226\left(56, \mathrm{M}^{+}\right), 211$ (100), 168 (14), 152 (11), 139 (21).

\section{2-(3-Pyridino)-benzophenone (3h)}

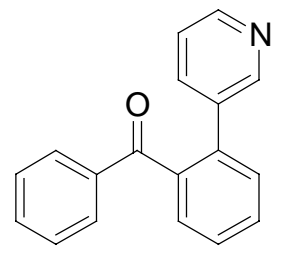

Prepared according to the TP 1 . To the $\mathrm{ZnBr}_{2}$ solution $(0.67 \mathrm{~mL}, 1.5 \mathrm{M}$ in THF) and NEP $(0.17 \mathrm{~mL})$ was added dropwise 3-pyridylmagnesium bromide $^{8}(1.57 \mathrm{~mL}, 0.83 \mathrm{M}$ in THF), 
then the catalyst solution $(0.08 \mathrm{~mL})$ and 2-bromobenzophenone $(270 \mathrm{mg}, 1.00 \mathrm{mmol})$. Stirred for $3 \mathrm{~h}$ at $50{ }^{\circ} \mathrm{C}$. The standard workup and purification by flash chromatography (pentan$\mathrm{CH}_{2} \mathrm{Cl}_{2}$ ) yielded $\mathbf{3 h}$ as a white solid $(197 \mathrm{mg}, 76 \%)$. The analytical data are in accordance with the literature. ${ }^{12}$

Mp.: $106-106.5^{\circ} \mathrm{C}$.

${ }^{1} \mathbf{H}-\mathbf{N M R}\left(\mathrm{CDCl}_{3}, 300 \mathrm{MHz}, 2{ }^{\circ} \mathrm{C}\right): 8.56-8.52(\mathrm{~m}, 1 \mathrm{H}), 8.44-8.40(\mathrm{~m}, 1 \mathrm{H}), 7.72-7.10(\mathrm{~m}$, $11 \mathrm{H})$.

${ }^{13} \mathbf{C}-\mathbf{N M R}\left(\mathrm{CDCl}_{3}, 75 \mathrm{MHz}, 25{ }^{\circ} \mathrm{C}\right): 198.3,149.8,148.8,139.5,137.9,137.6,136.6,136.3$, 133.6, 131.1, 130.6, 130.3, 129.5, 128.7, 128.2, 123.3 .

MS (70 eV, EI), m/z (\%): 77 (27), 105 (25), 127 (20), 182 (36), 230 (100), 231 (26), 259 (19, $\left.\mathrm{M}^{+}\right)$.

\section{5-(3-Fluorophenyl)-pyrimidine (3i)}

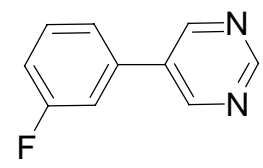

Prepared according to the TP 1 . To the $\mathrm{ZnBr}_{2}$ solution $(0.67 \mathrm{~mL}, 1.5 \mathrm{M}$ in THF) and NEP $(0.17 \mathrm{~mL})$ was added dropwise 3-fluorophenylmagnesium bromide $(1.57 \mathrm{~mL}, 0.83 \mathrm{M}$ in $\mathrm{THF})$, then the catalyst solution $(0.08 \mathrm{~mL})$ and 5-bromopyrimidine $(159 \mathrm{mg}, 1.00 \mathrm{mmol})$. Stirred for $1 \mathrm{~h}$ at RT. The standard work up and purification by flash chromatography (pentan-Et ${ }_{2} \mathrm{O}$ ) yielded $3 \mathbf{i}$ as a white solid (143 mg, 82\%).

Mp.: $63-63.5^{\circ} \mathrm{C}$.

${ }^{1}$ H-NMR $\left(\mathrm{CDCl}_{3}, 300 \mathrm{MHz}, 25^{\circ} \mathrm{C}\right): 9.13$ (s, 1H), 8.85 (s, 2H), 7.44-7.23 (m, 1H), 7.29-7.26 (m, 1H), 7.22-7.17 (m, 1H), 7.10-7.03 (m, 1H).

${ }^{13} \mathrm{C}-\mathrm{NMR}\left(\mathrm{CDCl}_{3}, 75 \mathrm{MHz}, 25{ }^{\circ} \mathrm{C}\right): 163.7(\mathrm{~d}, J=248 \mathrm{~Hz}), 158.3,155.2,136.8(\mathrm{~d}, J=7.9$ $\mathrm{Hz}), 133.5,131.5(\mathrm{~d}, J=8.5 \mathrm{~Hz}), 123.0,116.3(\mathrm{~d}, J=21.1 \mathrm{~Hz}), 114.3(\mathrm{~d}, J=21.1 \mathrm{~Hz})$.

IR (KBr): 2239 (w), 1591 (s), 1416 (s), 909 (vs), 734 (vs).

MS (70 eV, EI), m/z (\%): 94 (12), 105 (25), 120 (100), 173 (21), $174\left(96, \mathrm{M}^{+}\right)$.

HRMS $m / z$ : calcd. for $\mathrm{C}_{10} \mathrm{H}_{7} \mathrm{~N}_{2} \mathrm{~F}$ : 174.0593; found: 174.0577.

4-Pyrimidin-5-yl-benzoic acid ethyl ester (3j)

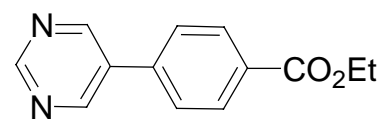

Prepared according to the TP 1 . To the $\mathrm{ZnBr}_{2}$ solution $(0.67 \mathrm{~mL}, 1.5 \mathrm{M}$ in THF) and NEP 
$(0.17 \mathrm{~mL})$ was added dropwise cold 4-carboethoxyphenylmagnesium bromide (prepared by iodine-magnesium exchange from ethyl 4-iodobenzoate and $i \mathrm{PrMgCl}-\mathrm{LiCl}^{8}$ at $-40{ }^{\circ} \mathrm{C}$ for 30 $\min )(1.57 \mathrm{~mL}, 0.83 \mathrm{M}$ in THF), then the catalyst solution $(0.08 \mathrm{~mL})$ in NEP), and 5bromopyrimidine $(159 \mathrm{mg}, 1.00 \mathrm{mmol})$. The mixture was stirred for $24 \mathrm{~h}$ at $\mathrm{RT}$, standard work up and purification by flash chromatography $\left(\mathrm{CH}_{2} \mathrm{Cl}_{2}\right.$-pentane) yielded $\mathbf{3 j}$ as pale yellow crystals (137 mg, 60\%). Analytical data are in accordance with literature. ${ }^{14}$

Mp.: $118-119^{\circ} \mathrm{C}$.

${ }^{1} \mathrm{H}-\mathrm{NMR}\left(\mathrm{CDCl}_{3}, 600 \mathrm{MHz}, 25{ }^{\circ} \mathrm{C}\right): \delta=9.25(\mathrm{~s}, 1 \mathrm{H}), 8.99(\mathrm{~s}, 2 \mathrm{H}), 8.19(\mathrm{~m}, 2 \mathrm{H}), 7.66(\mathrm{~m}$, $2 \mathrm{H}), 4.42(\mathrm{q}, J=7.2 \mathrm{~Hz}, 2 \mathrm{H}), 1.43(\mathrm{t}, J=7.2 \mathrm{~Hz}, 3 \mathrm{H})$.

${ }^{13} \mathrm{C}-\mathrm{NMR}\left(\mathrm{CDCl}_{3}, 150 \mathrm{MHz}, 25{ }^{\circ} \mathrm{C}\right): \delta=164.9,157.2,157.0,154.1,154.0,137.5,132.4$, $130.0,129.6,125.9,60.3,13.3$.

MS (70 eV, EI), m/z (\%): $228\left(21, \mathrm{M}^{+}\right), 200$ (33), 183 (100), 128 (40), 101 (32).

\section{8-(1-Naphthyl)-quinoline (3k)}

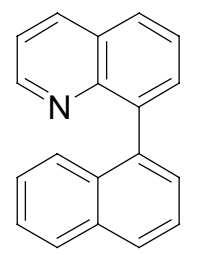

Prepared according to the TP 1 . To the $\mathrm{ZnBr}_{2}$ solution $(0.67 \mathrm{~mL}, 1.5 \mathrm{M}$ in THF) and NEP $(0.17 \mathrm{~mL})$ was added dropwise 1-naphthylmagnesium bromide $(1.57 \mathrm{~mL}, 0.83 \mathrm{M}$ in $\mathrm{THF})$, then the catalyst solution $(0.08 \mathrm{~mL})$ and 8 -quinolyl nonaflate ${ }^{13}(427 \mathrm{mg}, 1.00 \mathrm{mmol})$. Stirred for $24 \mathrm{~h}$ at RT. The standard workup and purification by flash chromatography $\left(\mathrm{CH}_{2} \mathrm{Cl}_{2}-\right.$ pentan) yielded 3k as a white solid (224 mg, $88 \%)$.

Мp.: $163-164^{\circ} \mathrm{C}$.

${ }^{1} \mathbf{H}-\mathbf{N M R}\left(\mathrm{CDCl}_{3}, 300 \mathrm{MHz}, 25^{\circ} \mathrm{C}\right)$ : 8.76-8.74 (m, 1H); 8.16-8.13 (m, 1H); 7.89-7.82 (m, $3 \mathrm{H})$; 7.69-7.66 (m, 1H), 7.60-7.46 (m, 3H), 7.41-7.18 (m, 4H).

${ }^{13} \mathrm{C}-\mathrm{NMR}\left(\mathrm{CDCl}_{3}, 75 \mathrm{MHz}, 25{ }^{\circ} \mathrm{C}\right): \delta=150.9,147.7,140.6,138.5,136.6,134.1,133.3$, 132.0, 128.9, 128.7, 128.5, 128.4, 128.3, 127.1, 126.6, 126.1, 126.0, 125.8, 121.5.

IR: (KBr) $\left(\mathrm{cm}^{-1}\right)$ : $3042(\mathrm{w}), 1593(\mathrm{w}), 1492$ (s), 829 (s), 797 (vs), 782 (vs), 773 (vs).

MS (70 eV, EI), m/z (\%): 127 (9), 226 (9), 252 (14), 254 (100), 255 (47, M+).

HRMS $m / z$ : calcd. for $\mathrm{C}_{19} \mathrm{H}_{13} \mathrm{~N}$ : 255.1048; found: 255.1020 . 
3-(1-Methyl-1H-pyrrol-2-yl)-pyridine (31).

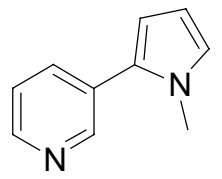

Prepared according to the TP 1 . To the $\mathrm{ZnBr}_{2}$ solution $(0.67 \mathrm{~mL}, 1.5 \mathrm{M}$ in THF) and NEP $(0.26 \mathrm{~mL})$ was added dropwise 1-methyl-2-pyrryllithium ${ }^{6}(2.4 \mathrm{~mL}, 0.5 \mathrm{M}$ in THF), then the catalyst solution $(0.08 \mathrm{~mL})$ and 3-bromopyridine $(158 \mathrm{mg}, 1.00 \mathrm{mmol})$. The reaction mixture was stirred for $22 \mathrm{~h}$ at $70{ }^{\circ} \mathrm{C}$, the standard workup and purification by flash chromatography $\left(\mathrm{Et}_{2} \mathrm{O}-\mathrm{CH}_{2} \mathrm{Cl}_{2}\right.$ 1:1) yielded $3 \mathrm{I}$ as yellow oil $(98 \mathrm{mg}, 62 \%)$. The analytical data are in accordance with the literature. ${ }^{7}$

${ }^{1} \mathbf{H}-\mathbf{N M R}\left(\mathrm{CDCl}_{3}, 300 \mathrm{MHz}, 25^{\circ} \mathrm{C}\right): \delta=8.66(\mathrm{~d}, J=1.8 \mathrm{~Hz}, 1 \mathrm{H}), 8.50\left(\mathrm{dd}, J_{1}=4.8 \mathrm{~Hz}, J_{2}=\right.$ $1.6 \mathrm{~Hz}, 1 \mathrm{H}), 7.70\left(\mathrm{ddd}, J_{1}=7.9 \mathrm{~Hz}, J_{2}=1.8 \mathrm{~Hz}, J_{3}=1.6 \mathrm{~Hz}, 1 \mathrm{H}\right), 7.31\left(\mathrm{ddd}, J_{1}=7.9 \mathrm{~Hz}, J_{2}=\right.$ $\left.4.8 \mathrm{~Hz}, J_{3}=0.8 \mathrm{~Hz}, 1 \mathrm{H}\right), 6.74\left(\mathrm{dd}, J_{1}=2.5 \mathrm{~Hz}, J_{2}=1.9 \mathrm{~Hz}, 1 \mathrm{H}\right), 6.27\left(\mathrm{dd}, J_{1}=3.6 \mathrm{~Hz}, J_{2}=\right.$ $1.9 \mathrm{~Hz}, 1 \mathrm{H}), 6.20\left(\mathrm{dd}, J_{1}=3.6 \mathrm{~Hz}, J_{2}=2.8 \mathrm{~Hz}, 1 \mathrm{H}\right), 3.65(\mathrm{~s}, 3 \mathrm{H})$.

${ }^{13} \mathbf{C}-\mathrm{NMR}\left(\mathrm{CDCl}_{3}, 75 \mathrm{MHz}, 25{ }^{\circ} \mathrm{C}\right): \delta=149.3,147.8,136.1,131.1,129.8,125.2,123.7$, $110.3,108.7,35.5$.

MS (70 eV, EI), m/z (\%): $158\left(100, \mathrm{M}^{+}\right), 143$ (7), 130 (19), 116 (6), 89 (5).

\section{1-(3'-Trifluoromethyl-biphenyl-4-yl)-ethanone (3m).}

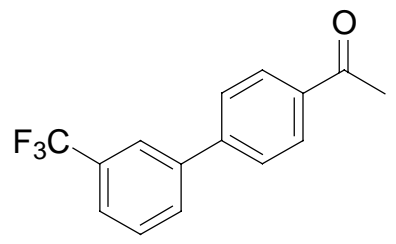

Prepared according to the TP 1 . To the $\mathrm{ZnBr}_{2}$ solution $(0.67 \mathrm{~mL}, 1.5 \mathrm{M}$ in THF) and NEP $(0.17 \mathrm{~mL})$ was added dropwise 3-trifluoromethyl-phenyl-magnesium bromide ${ }^{8}(1.57 \mathrm{~mL}, 0.83$ $\mathrm{M}$ in THF), then the catalyst solution $(0.08 \mathrm{~mL})$ and 4-bromoacetophenone (199 $\mathrm{mg}$, $1.00 \mathrm{mmol}$ ) Stirred for $18 \mathrm{~h}$ at RT, the standard workup and purification by flash chromatography $\left(\mathrm{CH}_{2} \mathrm{Cl}_{2}\right.$-pentane 1:1) yielded $\mathbf{3 m}$ as colourless oil (180 $\left.\mathrm{mg}, 68 \%\right)$. The 
analytical data are in accordance with the literature. ${ }^{9}$

${ }^{1} \mathbf{H}-\mathbf{N M R}\left(\mathrm{CDCl}_{3}, 300 \mathrm{MHz}, 25{ }^{\circ} \mathrm{C}\right): \delta=8.06\left(\mathrm{ddd}, J_{1}=8.6 \mathrm{~Hz}, J_{2}=2.4 \mathrm{~Hz}, J_{3}=2.0 \mathrm{~Hz}, 2 \mathrm{H}\right)$, 7.87-7.78 (m, 2H), 7.73-7.57 (m, 4H), $2.65(\mathrm{~s}, 3 \mathrm{H})$.

${ }^{13} \mathrm{C}-\mathrm{NMR}\left(\mathrm{CDCl}_{3}, 75 \mathrm{MHz}, 25{ }^{\circ} \mathrm{C}\right): \delta=197.9,144.6,141.1,136.9,132.2,132.0,131.6$, $130.9,130.2,129.9,129.5,127.4,125.3,124.4,27.1$.

MS (70 eV, EI), m/z (\%): 264 (35, M+), 249 (100), 221 (6), 201 (34), 152 (21).

\section{Ethyl 4-(1,3-benzodioxol-5-yl)benzoate (3n)}

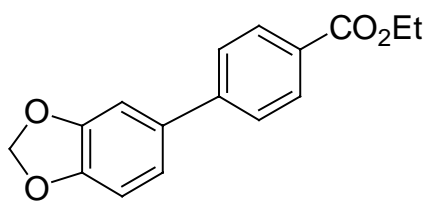

Prepared according to the TP 1 . To the $\mathrm{ZnBr}_{2}$ solution $(0.67 \mathrm{~mL}, 1.5 \mathrm{M}$ in THF) and NEP $(0.17 \mathrm{~mL})$ was added dropwise 1,3-benzodioxol-5-yl-magnesium bromide $(1.57 \mathrm{~mL}, 0.83 \mathrm{M}$ in THF), then the catalyst solution $(0.08 \mathrm{~mL})$ and ethyl 4-bromobenzoate $(229 \mathrm{mg}$, $1.00 \mathrm{mmol})$. Stirred for $5 \mathrm{~h}$ at RT. The usual workup and purification by flash chromatography (pentane-ether 1:1) cromatography yielded 3n as white solid (253 mg, 94\%). Mp.: $92.5-93.5^{\circ} \mathrm{C}$.

${ }^{1}$ H-NMR $\left(\mathrm{CDCl}_{3}, 300 \mathrm{MHz}, 25^{\circ} \mathrm{C}\right): \delta=8.07(\mathrm{~d}, J=8.7 \mathrm{~Hz}, 2 \mathrm{H}), 7.56(\mathrm{~d}, J=8.7 \mathrm{~Hz}, 2 \mathrm{H})$, 7.11-7.07 (m, 2H), 6.89 (d, $J=8.6 \mathrm{~Hz}, 1 \mathrm{H}), 5.00(\mathrm{~s}, 2 \mathrm{H}), 4.39(\mathrm{q}, J=7.1 \mathrm{~Hz}, 2 \mathrm{H}), 1.40(\mathrm{~d}, J=$ $7.2 \mathrm{~Hz}, 3 \mathrm{H})$.

${ }^{13} \mathrm{C}-\mathrm{NMR}\left(\mathrm{CDCl}_{3}, 75 \mathrm{MHz}, 25{ }^{\circ} \mathrm{C}\right): \delta=166.4,148.3,147.7,145.1,134.3,130.0,128.8$, 126.6, 121.0, 108.6, 107.6, 101.3, 60.9, 14.3.

IR (KBr): 2904 (w), 1707 (vs), 1606 (m), 1522 (w), 1503 (m), 1486 (s), 1410 (s), 1274 (vs), 1256 (s), 1235 (m), 1182 (s), 1107 (s), 1036 (s), 932 (m), 858 (m), 772 (s), 702 (w).

MS (70 eV, EI), m/z (\%): 270 (100, M+), 242 (32), 225 (70), 139 (40), 112 (5), 63 (2).

HRMS $m / z$ : calcd. for $\mathrm{C}_{16} \mathrm{H}_{14} \mathrm{O}_{4}: 270.0892$; found: 270.0888 . 


\section{3-(1,3-benzodioxol-5-yl)pyridine (3o)}

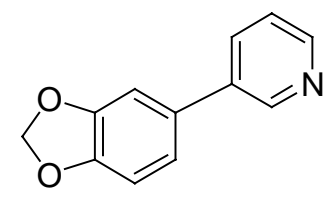

Prepared according to the TP 1 . To the $\mathrm{ZnBr}_{2}$ solution $(0.67 \mathrm{~mL}, 1.5 \mathrm{M}$ in THF) and NEP $(0.17 \mathrm{~mL})$ was added dropwise 1,3-benzodioxol-5-yl-magnesium bromide $(1.57 \mathrm{~mL}, 0.83 \mathrm{M}$ in THF), then the catalyst solution (0.08 $\mathrm{mL}$ in NEP) and 3-bromopyridine (158 $\mathrm{mg}, 1.00$ mmol). Stirred for $5 \mathrm{~h}$ at RT. The standard workup and purification by flash chromatography yielded 30 as a white solid (165 mg, $83 \%)$.

Mp.: $92-92.5^{\circ} \mathrm{C}$.

${ }^{1} \mathrm{H}-\mathrm{NMR}\left(\mathrm{CDCl}_{3}, 300 \mathrm{MHz}, 25^{\circ} \mathrm{C}\right): \delta=8.76(\mathrm{~d}, J=1.9 \mathrm{~Hz}, 1 \mathrm{H}), 8.54-8.51(\mathrm{~m}, 1 \mathrm{H}), 7.78$ $7.74(\mathrm{~m}, 1 \mathrm{H})$, 7.32-7.27 (m, 1H), 7.04-7.00 (m, 2H), 6.90-6.87 (m, 1H), $5.99(\mathrm{~s}, 2 \mathrm{H})$.

${ }^{13} \mathrm{C}-\mathrm{NMR}\left(\mathrm{CDCl}_{3}, 75 \mathrm{MHz}, 25{ }^{\circ} \mathrm{C}\right): \delta=148.4,148.1,148.0,147.7,136.3,133.9,131.9$, $123.4,120.8,108.8,107.5,101.3$.

IR (KBr): 2912 (w), 1512 (s), 1479 (vs), 1420 (s), 1294 (w), 1266 (m), 1238 (s), 1111 (w), 1037 (s), 931 (m), 806 (s), 706 (m).

MS (70 eV, EI), m/z (\%): $199\left(100, \mathrm{M}^{+}\right), 140$ (10), 114 (11), 88 (4), 63 (3).

HRMS $m / z$ : calcd. for $\mathrm{C}_{12} \mathrm{H}_{9} \mathrm{NO}_{2}$ : 199.0633; found: 199.0602 .

\section{1-(3,4-methylenedioxyphenyl)-naphthalene (3p)}

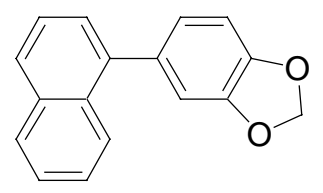

Prepared according to the TP 1. To $\mathrm{ZnBr}_{2}$ or $\mathrm{ZnCl}_{2}$ solution $(0.67 \mathrm{~mL}, 1.5 \mathrm{M}$ in THF) and NEP $(0.17 \mathrm{~mL})$ was added dropwise 1-naphthylmagnesium bromide $(1.57 \mathrm{~mL}, 0.83 \mathrm{M}$ in $\mathrm{THF})$, then the catalyst solution $(0.08 \mathrm{~mL})$ and 3,4-methylenedioxyphenyl triflate ${ }^{10}(270 \mathrm{mg}$, $1.00 \mathrm{mmol}$ ). Stirred for $24 \mathrm{~h}$ at RT. The standard workup and purification by flash chromatography (pentane-Et ${ }_{2} \mathrm{O}$ 9:1) yielded 3p as colourless oil (196 mg, 79\%, for the reaction with $\mathrm{ZnCl}_{2} 77 \%$ yield). The analytical data are in accordance with the literature. ${ }^{11}$

${ }^{1} \mathbf{H}-\mathbf{N M R}\left(\mathrm{CDCl}_{3}, 300 \mathrm{MHz}, 2{ }^{\circ} \mathrm{C}\right): \delta=7.99-7.85(\mathrm{~m}, 3 \mathrm{H}), 7.55-7.41(\mathrm{~m}, 4 \mathrm{H})$, 7.02-6.94 (m, 
$3 \mathrm{H}), 6.06(\mathrm{~s}, 2 \mathrm{H})$.

${ }^{13} \mathrm{C}-\mathrm{NMR}\left(\mathrm{CDCl}_{3}, 75 \mathrm{MHz}, 25{ }^{\circ} \mathrm{C}\right): \delta=147.9,174.3,140.2,135.1,134.2,132.2,128.7$, $128.0,127.3,126.4,126.2,125.8,123.8,111.1,108.6,101.5$.

MS (70 eV, EI), m/z (\%): $248\left(100, \mathrm{M}^{+}\right), 217$ (19), 208 (10), 189 (52), 94 (20).

\section{References:}

1. Lourak, M.; Vanderesse, R.; Fort, Y.; Caubere, P. J. Org. Chem. 1989, 54, 4844.

2. Andrus, M. B. ; Song, C. Org. Lett. 2001, 3, 3761.

3. Nakao, Y.; Oda, T.; Sahoo, A. K.; Hiyama, T. J. Organomet. Chem. 2003, 687(2), 570.

4. Cioffi, C. L.; Spencer, W. T.; Richards, J.; Herr, R. J. J. Org. Chem. 2004, 69, 2210.

5. Hatanaka, Y.; Goda, K.; Yoshinori, O.; Hiyama, T. Tetrahedron 1994, 50, 8301.

6. Brittain, J. M.; Jones, R. A.; Arques, J. S.; Saliente, T. A. Synth. Comm. 1982, 12, 231.

7. Baxendale, I. ; Brusotti, M.; Ley, S. J. Chem. Soc. Perkin 1, 2002, 143.

8. Krasovskiy, A.; Knochel, P. Angew. Chem. Int. Ed. 2004, 3333.

9. Solodenko, W.; Schön, U.; Messinger, J.; Glinschert, A.; Kirschning, A. Synlett 2004, 10, 1699.

10. Echavarren, A. M.; Stille, J. K. J. Am. Chem. Soc. 1987, 109, 5478.

11. Shimada, S.; Yamazaki, O.; Toshifumi, T.; Rao, M.; Suzuki, Y.; Tanaka, M. Angew. Chem. Int. Ed. 2003, 42, 1845.

12. Edwards, M. L.; Stemerick, D. M; Diekema, K. A.; Dienerstein, R. J. J. Med. Chem. 1994, $37,4357$.

13. Subramanian, L. R.; Garcia Martinez, A.; Herrera Fernandez, A.; Martinez Alvarez, R. Synthesis, 1984, 6, 481.

14. Kano, S.; Yuasa, Y.; Shibuya, S. ; Hibino, S. Heterocycles, 1982, 19, 1079. 

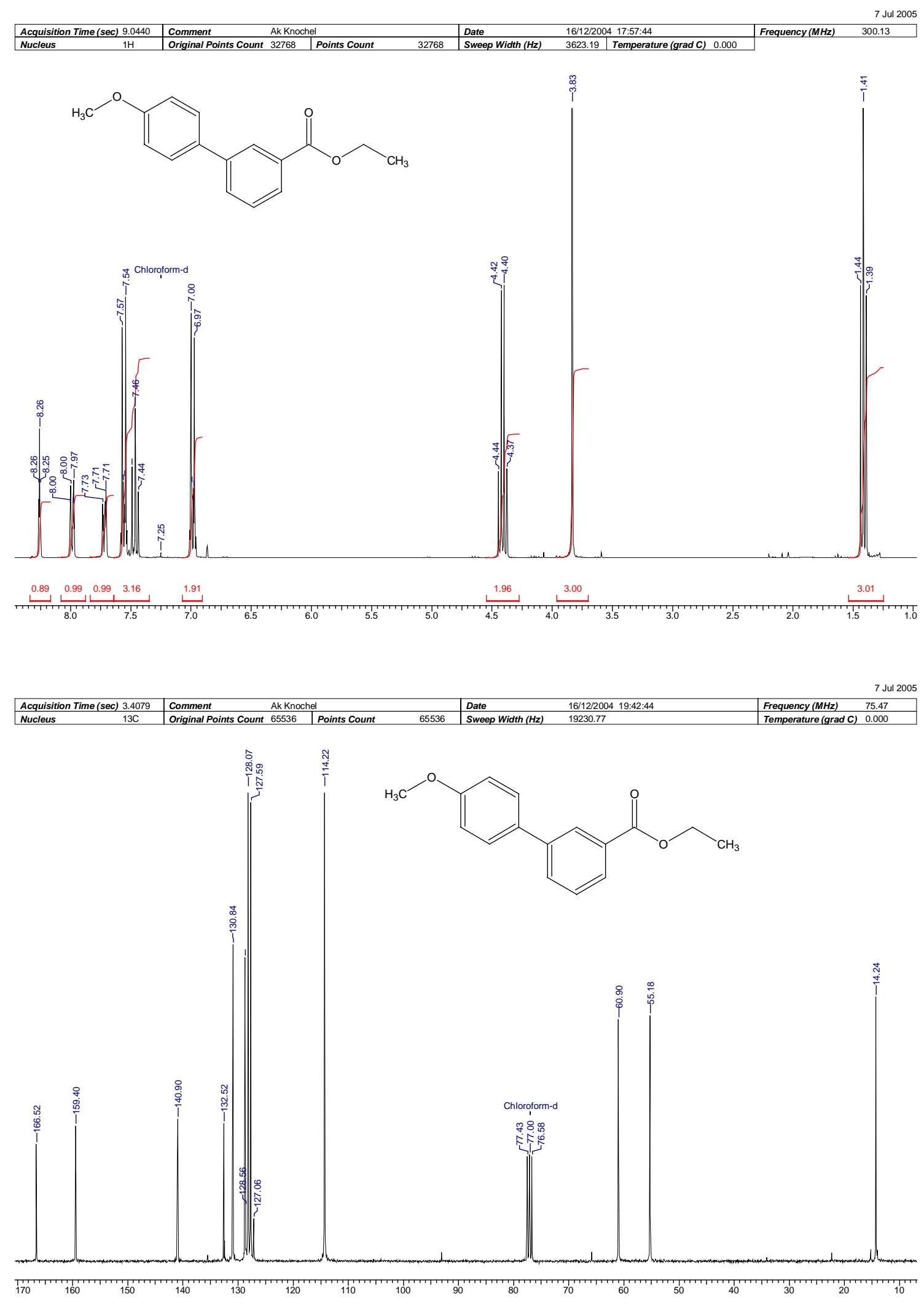
S13
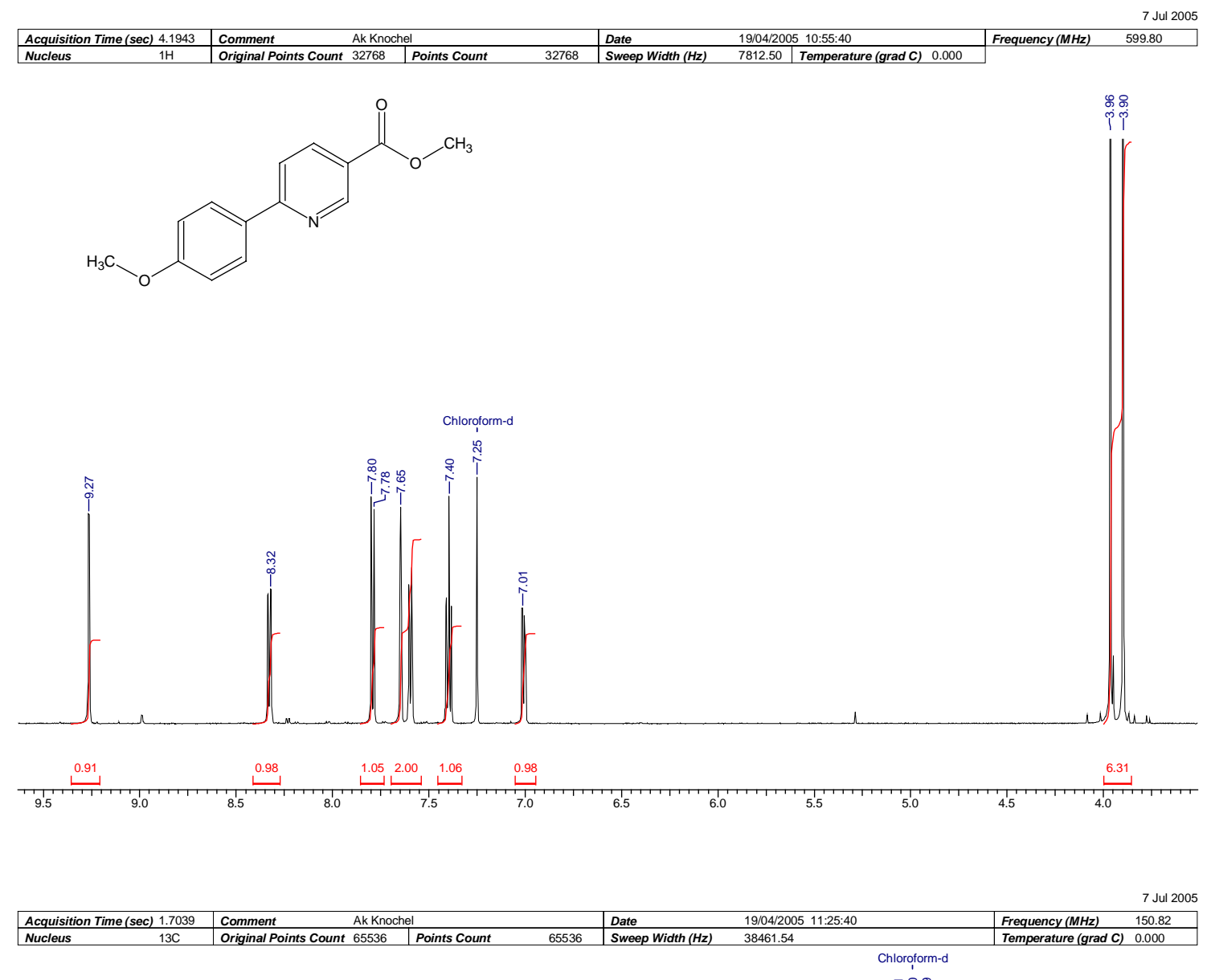<smiles>COC(=O)c1ccc(-c2ccc(OC)cc2)nc1</smiles>

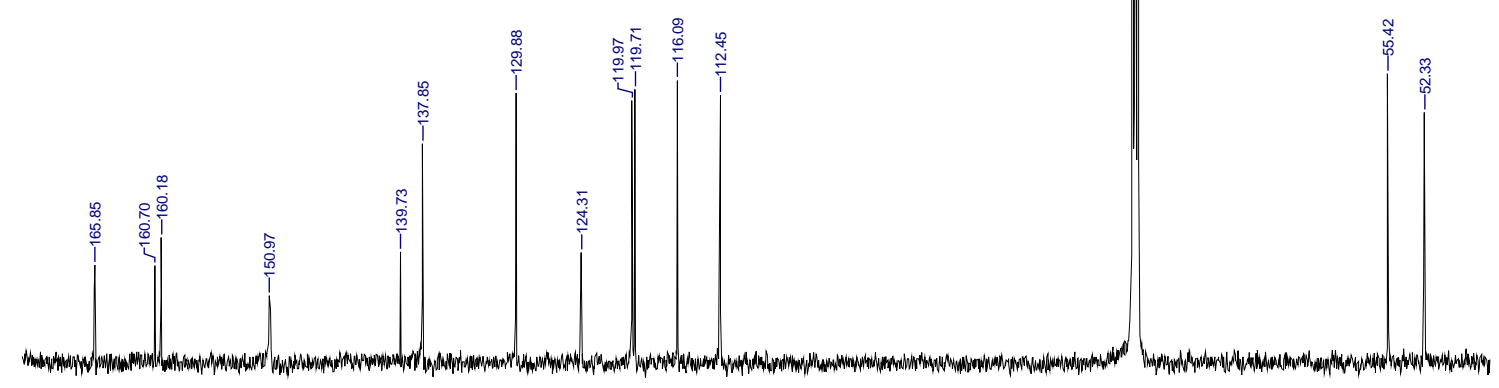


S14
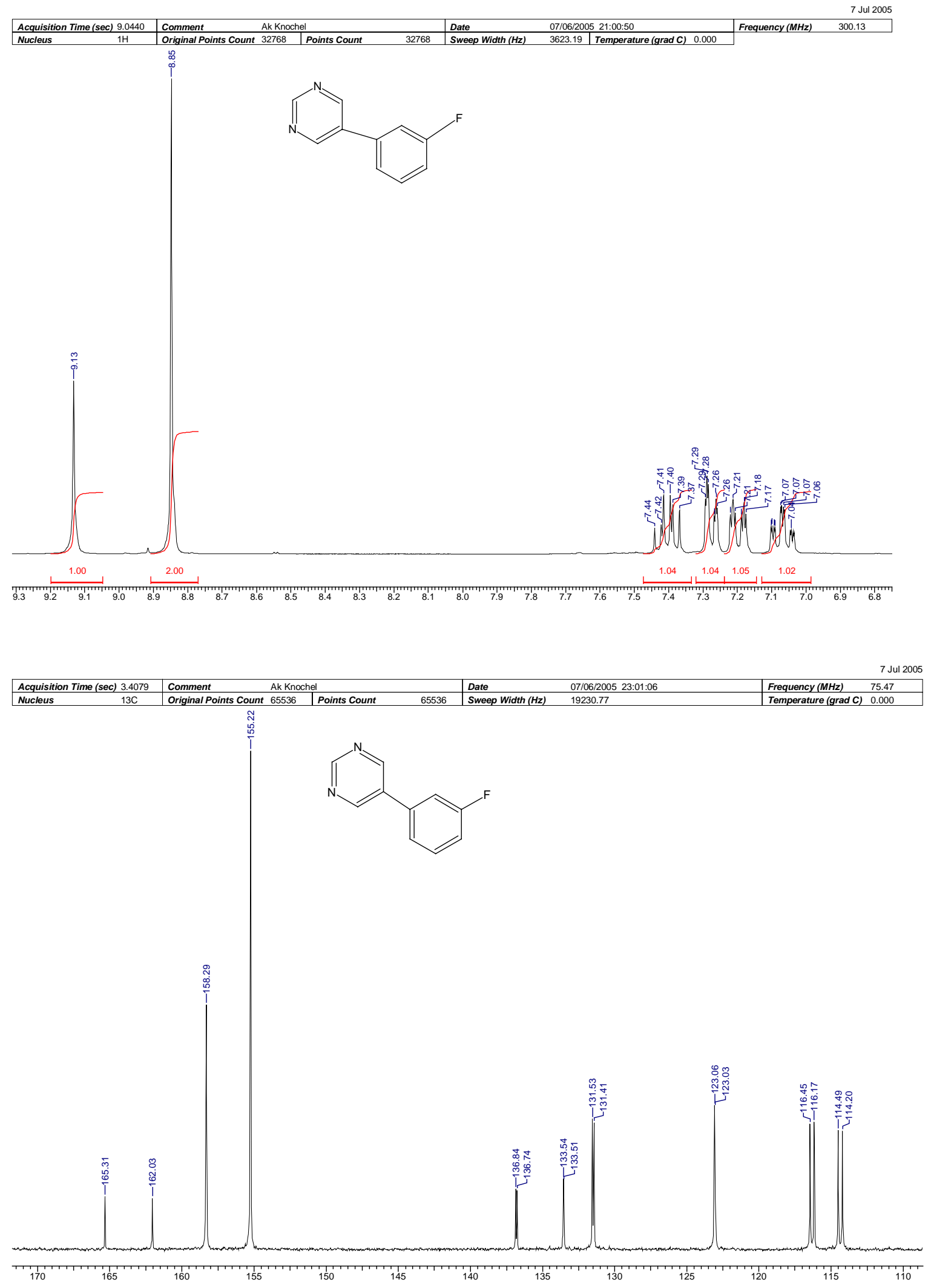
S15
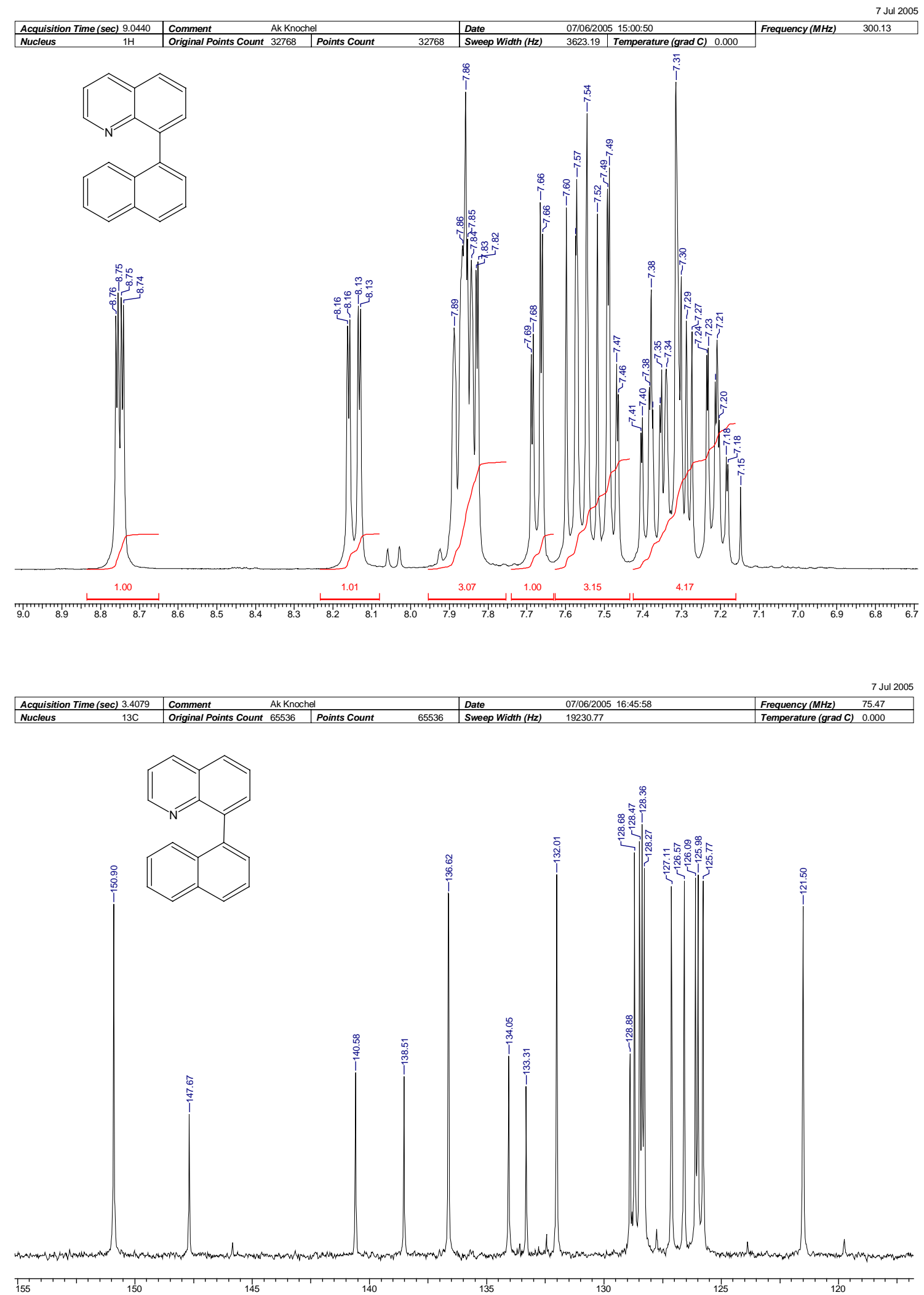
S16
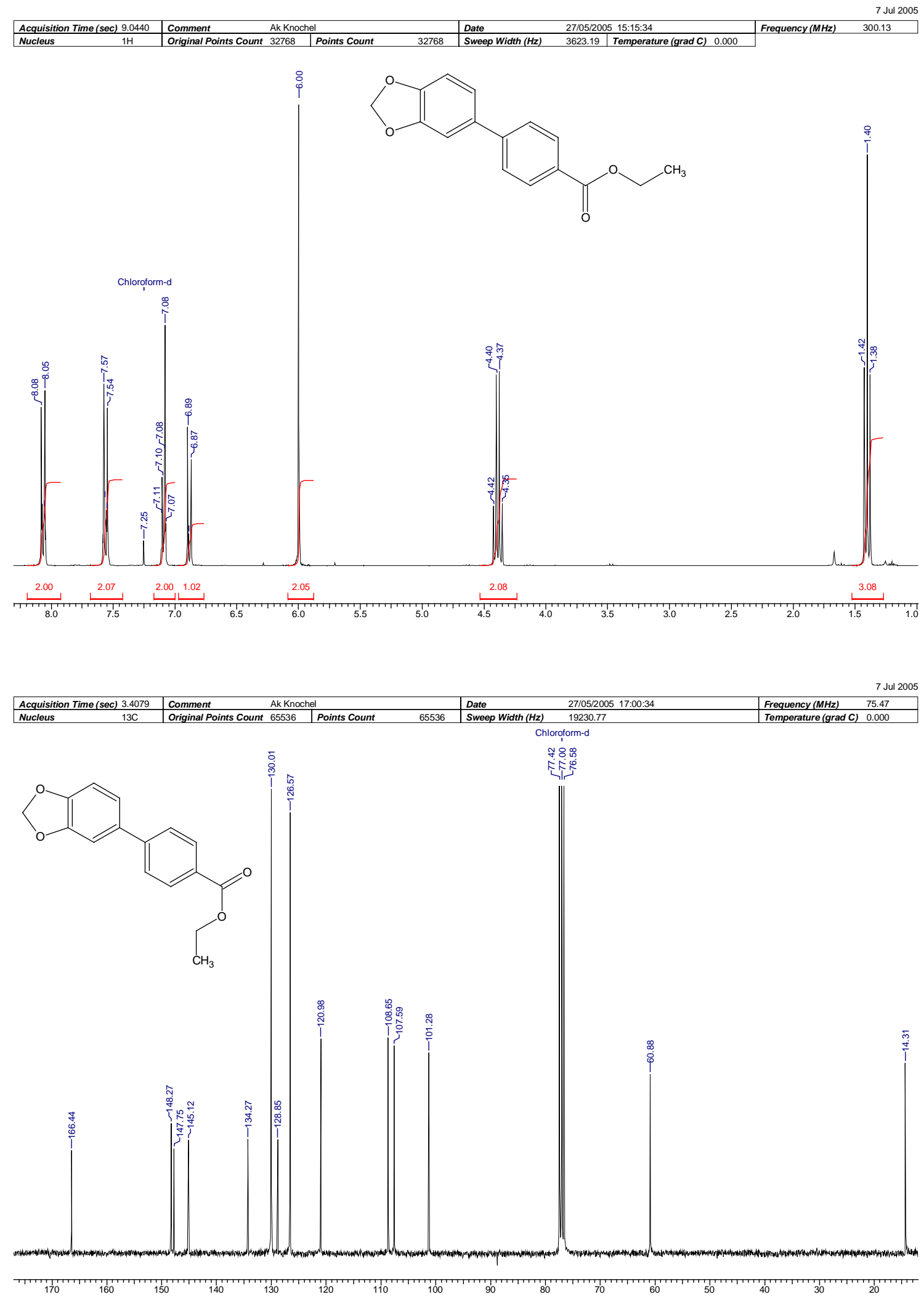


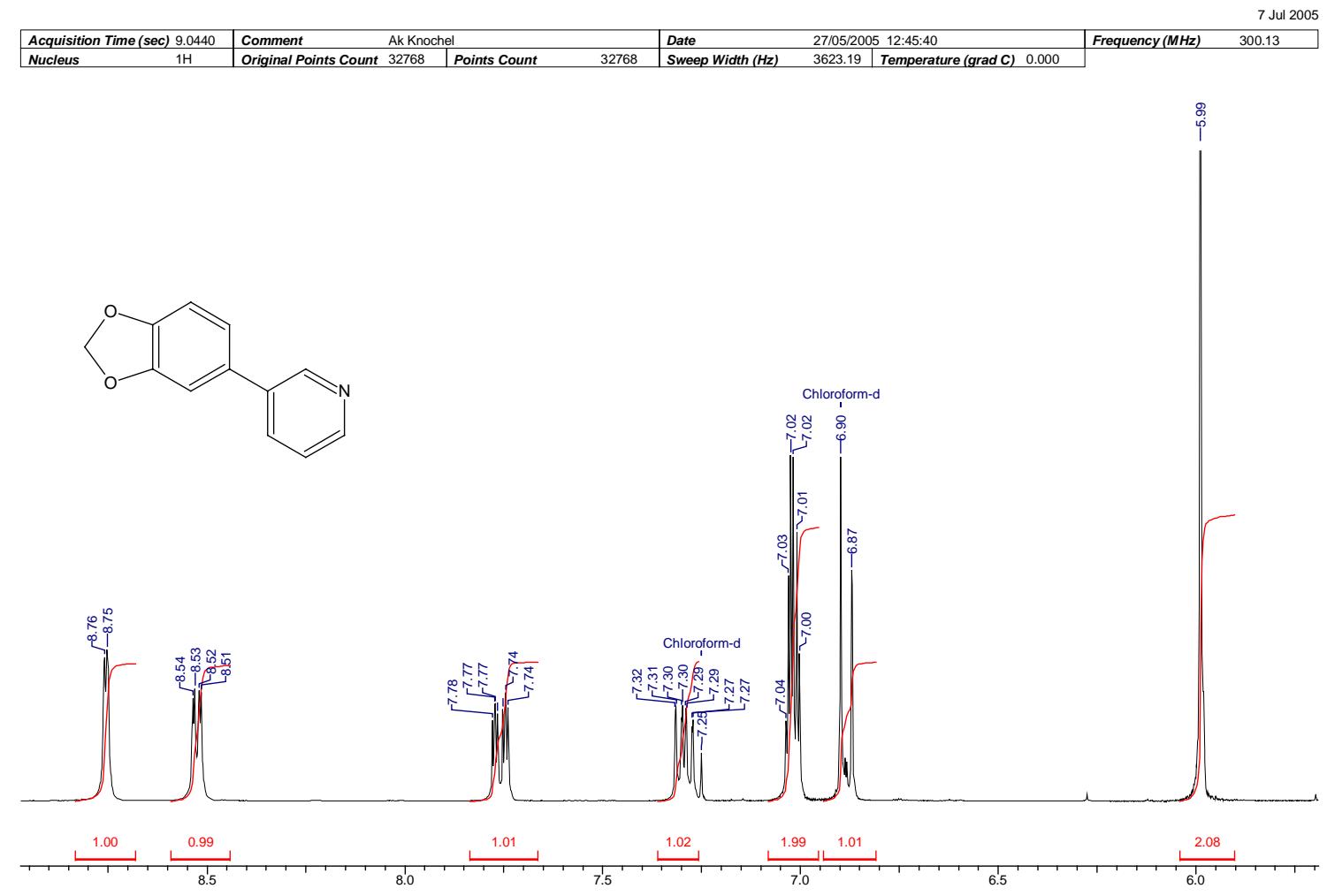

$x$ 


\section{References:}

1. Lourak, M.; Vanderesse, R.; Fort, Y.; Caubere, P. J. Org. Chem. 1989, 54, 4844.

2. Andrus, M. B. ; Song, C. Org. Lett. 2001, 3, 3761.

3. Nakao, Y.; Oda, T.; Sahoo, A. K.; Hiyama, T. J. Organomet. Chem. 2003, 687(2), 570.

4. Cioffi, C. L.; Spencer, W. T.; Richards, J.; Herr, R. J. J. Org. Chem. 2004, 69, 2210.

5. Hatanaka, Y.; Goda, K.; Yoshinori, O.; Hiyama, T. Tetrahedron 1994, 50, 8301.

6. Brittain, J. M.; Jones, R. A.; Arques, J. S.; Saliente, T. A. Synth. Comm. 1982, 12, 231.

7. Baxendale, I. ; Brusotti, M.; Ley, S. J. Chem. Soc. Perkin 1, 2002, 143.

8. Krasovskiy, A.; Knochel, P. Angew. Chem. Int. Ed. 2004, 3333.

9. Solodenko, W.; Schön, U.; Messinger, J.; Glinschert, A.; Kirschning, A. Synlett 2004, 10, 1699.

10. Echavarren, A. M.; Stille, J. K. J. Am. Chem. Soc. 1987, 109, 5478.

11. Shimada, S.; Yamazaki, O.; Toshifumi, T.; Rao, M.; Suzuki, Y.; Tanaka, M. Angew. Chem. Int. Ed. 2003, 42, 1845.

12. Edwards, M. L.; Stemerick, D. M; Diekema, K. A.; Dienerstein, R. J. J. Med. Chem. 1994, $37,4357$.

13. Subramanian, L. R.; Garcia Martinez, A.; Herrera Fernandez, A.; Martinez Alvarez, R. Synthesis, 1984, 6, 481.

14. Kano, S.; Yuasa, Y.; Shibuya, S. ; Hibino, S. Heterocycles, 1982, 19, 1079. 